\title{
Efficacy of anti-HER2 therapy in metastatic breast cancer by discordance of HER2 expression between primary and metastatic breast cancer.
}

Elisa Van Raemdonck ${ }^{1}$, Floris $\mathrm{G}^{3}$ Berteloot $\mathrm{P}^{1}$, Laenen $\mathrm{A}^{2}$,Vergote $\mathrm{I}^{1}$, Floris $\mathrm{G}^{3}$, Wildiers $\mathrm{H}^{4}$, Punie $\mathrm{K}^{4}$, Neven $\mathrm{P}^{1}$

\section{Author information}

Affiliations

1 KU Leuven - University of Leuven, Department of Obstetrics and Gynecology, Herestraat 49, B-3000 Leuven, Belgium

2 KU Leuven - University of Leuven, Department of Biostatistics, Herestraat 49, B-3000 Leuven, Belgium 3 KU Leuven - University of Leuven, Department of Imaging and Pathology, Laboratory of Translational Cell \& Tissue Research and University Hospitals Leuven, Department of Pathology, Herestraat 49, B-3000 Leuven, Belgium

4 KU Leuven - University of Leuven, Department of General Medical Oncology, Herestraat 49, B-3000 Leuven, Belgium

Corresponding authors:

Neven Patrick

Van Raemdonck Elisa

Mail:

Patrick.neven@uzleuven.be

Elisa.vanraemdonck@student.kuleuven.be 


\begin{abstract}
Purpose:

In stage IV breast cancer, the efficacy of human epidermal growth factor receptor 2 (HER2) targeted therapies in cases with discordance in HER2 expression between primary and metastatic site is not well known. We studied progression free (PFS) and overall survival (OS) by HER2 concordance when treating women with taxanetrastuzumab (+/-pertuzumab) in first or second line and trastuzumab-emtansine (T-DM1) or capecitabine-lapatinib in later lines.
\end{abstract}

\title{
Patients and Methods:
}

Retrospective monocentric study including all breast cancer patients receiving trastuzumab between January 2002 and September 2017 at the University Hospital in Leuven; we selected metastatic patients with an available HER2 status in primary and metastatic site. The Kaplan-Meier method was used for estimating PFS/OS and log-rank test for analyzing between group differences. A Cox model is used for testing difference between groups while correcting for Pertuzumab. Multivariable Cox regression is used to model overall survival as a function group, correcting for possible confounders.

\section{Results:}

We included 74 patients; 46 had an unchanged HER2 status (positive/positive), 9 lost HER2 (positive/negative), while 19 acquired HER2 amplification (negative/positive). 25 out of 28 cases with a discordant HER2 status were positive for ER and/or PgR in the primary site. HER2 positive/negative cases had a significantly lower PFS for taxane-trastuzumab-(pertuzumab) (PFS = 5.5 months), compared to HER2 positive/positive (PFS 9 months, $\mathrm{p}=$ $0.01)$ and HER2 negative/positive (PFS 14 months, $\mathrm{p}=0.01)$ patients. PFS for later line T-DM1 ( $\mathrm{n}=30)$ was significantly higher for the HER2 positive/positive group (PFS 6.0 months) than for the discordant groups HER2 negative/positive (PFS 1.0 month, $\mathrm{p}=0.04$ ) and HER2 positive/negative (PFS 1.5 month, $\mathrm{p}=0.01$ ). After correcting for possible confounders, the HER2 positive/negative group had a significantly worse OS compared to HER2 positive/positive (HR 0.19, 95\% CI $0.08-0.44$ ) and negative/positive (HR 0.15, 95\% 0.06-0.38).

\section{Conclusion:}

Conversion of HER2 status was seen in 28 out of 74 cases and was mostly observed in hormone receptorpositive tumors. In contrast to patients with HER2 loss, patients with a positive conversion of HER2 status derived substantial benefit from first line treatment with taxane-trastuzumab-(pertuzumab). This study highlights the importance of re-biopsying the metastatic lesion and changing treatment according to the last HER2 result.

\section{Keywords}

Discordance, HER2 receptor, breast cancer, metastasis, biopsy, survival, HER2/CEP17 ratio.

\begin{tabular}{|c|c|c|c|}
\hline \multicolumn{4}{|c|}{ Abbreviations } \\
\hline $\mathrm{BC}$ & Breast cancer & ER & Estrogen receptor \\
\hline PgR & Progesterone receptor & OS & Overall survival \\
\hline CEP17 & Centromere enumerator probe 17 & PFS & Progression free survival \\
\hline TTM & Time to metastasis & HER2 & Human epidermal growth factor receptor 2 \\
\hline NPI & Nottingham Prognostic Index & $\mathrm{CI}$ & Confidence intervals \\
\hline
\end{tabular}

\section{Introduction}

The Human Epidermal Growth factor receptor 2 gene (HER2) is an oncogene encoding for a transmembrane receptor kinase of the epidermal growth factor family. HER2 amplification leads usually to protein overexpression which is observed in about $15 \%$ of primary breast cancers and is historically associated to higher metastatic potential, and an adverse prognosis with a decreased overall survival. (13) The currently approved anti-HER2 therapies (trastuzumab, pertuzumab, T-DM1, and HER2 kinase 
inhibitors) have profoundly improved the survival outcome of HER2 positive breast cancers. (4) In current practice, the receptor status of the biopsy of the primary breast cancer is mainly used to direct the therapeutic management of patients with metastatic breast cancer. However, biomarker characteristics of the primary tumor may change throughout tumor progression from the primary breast cancer to the metastatic lesions. (3,5-7) A meta-analysis of Aurilio et al., including 48 studies from the last two decades, reported pooled discordance rates of $20 \%$ for estrogen receptor (ER), $33 \%$ for progesterone receptor $(\mathrm{PgR})$ and $8 \%$ for HER2 receptor. (8) A meta-analysis of Schrijver et al., including 39 studies, found positive to negative conversion for HER2 status in $21.3 \%$ and negative to positive conversion in $9.5 \%$. (9)

Therefore, the American Society of Clinical Oncology/College of American Pathologist (ASCO/CAP) guidelines of 2015 recommended that in patients with accessible metastases, biopsy for confirmation and retesting of ER, PgR and HER2 should be offered. However, if discordances are found, evidence is still lacking to determine whether outcomes are better with treatment based on receptor status in the metastases or the primary tumor. (10) Notably, a pooled analysis of Amir et al., has shown that biopsy of recurrent disease was associated with a reported change in choice of therapy in $14 \%$ of women; suggesting clinicians would modify their choice of therapy only once for every seven biopsies of recurrent disease performed. $(5,6,11)$

Retrospective studies of primary and metastatic breast cancer suggested that receptor discordance is associated with a poorer outcome, presumably due to use of inappropriate therapy or the selection of tumors with a more unstable phenotype and therefore more aggressive behavior. $(5,7,12,13)$ However, the DESTINY study showed that discordance between primary and metastatic lesion was not associated with apparent differences in overall survival if treatment was modified accordingly. (5)

In this retrospective study, we hypothesized that patients benefit less of anti-HER2 therapy if HER2 amplification was lost in the metastatic lesion. We therefore determined the concordance rate in HER2 status between primary and metastatic breast cancer in consecutive women with stage IV breast cancer who received trastuzumab and had a meta-/synchronous biopsy of primary breast and metastatic lesion. The primary objective was to evaluate efficacy of HER2 inhibitors dependent on HER2 concordance between primary and metastatic breast cancer tissue.

\section{Methods}

\section{Study population and materials}

We retrospectively selected all consecutive cases from our institutional database who received trastuzumab for breast cancer in a (neo-) adjuvant or metastatic setting between January 2002 and October 2017. Women with a synchronous (primary) and with a metachronous (secondary) metastatic breast cancer were included. Only patients with biopsy of primary and metastatic lesion and 
determination of HER2 status on both biopsies were selected for our study. The timing of the biopsy of the metastatic disease was not taken into account. We excluded women with a non-metastatic disease, patients with a bilateral breast carcinoma with a discordant HER2 status, patients without biopsy of the metastatic disease and without determination of the HER2 status on the primary and metastatic lesion. We annotated the exact moment that the biopsy of the metastatic lesion was taken, and the location of the biopsy of the metastasis was reported.

The HER 2 status was derived from the pathological report and not centrally reassessed. Pathological reports were collected from five different centers, but in most cases from our own hospital. HER2 analysis was performed using either standard immunohistochemistry (IHC) and fluorescent in situ hybridization (FISH). In Belgium, all IHC 2+ and 3+ receive FISH testing since trastuzumab is only reimbursed in case of confirmed HER2 amplification by in situ hybridization (ISH). In the past, many centers were used to perform ISH on all cases, because ISH testing was always reimbursed.

For interpretation of HER2 testing the most recent ASCO/CAP guidelines at the time of diagnosis were used. During the period between 2011 and 2017 there have been two updates. A positive ISH test was considered as a HER2/CEP17 above 2.0 or more than 6 HER 2 signals per cell. In case of heterogeneity samples analysed in University Hospital UZ Leuven reported the percentage of tumor cells with enhanced number of HER2 signals by ISH or the proportion of tumor cells with a ratio above 2 . Heterogeneity in staining pattern by IHC was reported as well and annotated.

In the University Hospital UZ Leuven, treatment with trastuzumab was introduced in 2007, cases diagnosed before this time had no treatment with trastuzumab in (neo-)adjuvant setting.

The most common reasons to perform a biopsy were a poor therapeutic response and inclusion in clinical trials. In patients with a metachronous metastatic breast cancer the second biopsy was mostly taken before the start of the therapy in metastatic setting. In cases with a synchronous metastatic disease the second biopsy was mostly taken after at least one therapeutic line, in a few cases it was taken before the start of treatment.

We collected the start and stop date of different treatment lines. In patients with metachronous metastatic disease we annotated the time to metastasis (TTM) and Nottingham prognostic index (NPI) score. For all cases status of hormone receptors and TNM classification of the primary breast tumor was noted.

For calculating the progression free survival of taxane and trastuzumab (+/- pertuzumab) we only included patients who received this treatment in first or second line. No restriction was made on number of previous lines for T-DM1 and lapatinib with capecitabine.

\section{Objective and end points}


The primary objective of this retrospective study was to evaluate the predictive value of change of HER2 status for progression free survival using HER2 inhibitors (concomitant with chemotherapy) in the metastatic setting. The following therapies for stage IV disease were examined for PFS: a taxane with trastuzumab (+/- pertuzumab), T-DM1 or capecitabine with lapatinib. The secondary objective was to make a descriptive analysis of the general characteristics of patients with a HER2 switch.

The primary endpoint of this study was the progression free survival (PFS). PFS was considered as the period elapsing from the initiation of therapy to the end of therapy because of progressive disease or death. Not all patients that received taxane and trastuzumab had concomitant therapy with pertuzumab, therefore we corrected for pertuzumab use. Furthermore, we compared OS in patients dependent on the dynamic HER2 status. We corrected OS for possible confounders like presence of primary metastatic disease, negative sex-steroid receptor in the primary tumor, a high NPI score (> 5.4) and a short TTM ( $\leq 12$ months). OS was defined as the duration between the start of therapy in metastatic setting to death as result of any cause.

\section{Statistical analysis}

The Kaplan-Meier method was used for estimating PFS and OS. For calculation of PFS, subgroups were compared using the log-rank statistic. We used a cox model for testing the difference between groups while correcting for pertuzumab. Cox regression was used to model OS as a function group, correcting for possible confounders (presence of primary metastatic disease, negative sex-steroid receptor in the primary tumor, a high NPI score ( $>5.4)$ and a short TTM ( $\leq 12$ months)), and to model PFS as a function of the HER2/CEP17 ratio at the biopsy of the primary lesion and of the metastatic lesion.

Results were presented as hazard ratios (HR) with a 95\% confidence interval. Two-sided tests with Pvalues less than 0.05 were considered statistically significant. Analysis has been performed using SAS software (version 9.4 of the SAS System for Windows).

\section{Results}

In the University Hospital of Leuven, 1100 patients had a treatment with trastuzumab for breast cancer in the period between January 2002 and September 2017. Out of this population, 74 patients had a synchronous or metachronous metastatic disease and a HER2 status performed on the biopsy of the primary and metastatic breast cancer. Metastatic disease was synchronous in 22 patients and metachronous in 52 patients. (Table 1) A CONSORT flow diagram is shown in figure 1.

\section{Patient characteristics}

Patients demographics and tumor characteristics are summarised in table 1. 
In our population of 74 selected patients, 9 out of 55 cases with a positive HER2 status in the primary breast tumor had a negative HER2 status in the metastatic tumor, a conversion rate of $16.4 \%$. The remaining 19 cases had a positive conversion of the HER2 status between the primary breast and metastatic lesions. In the 52 patients with metachronous metastatic disease, 19 patients had a discordance in HER2 status. Six patients switched from HER2 positive in the primary tumor to HER2 negative status in the metastatic lesion (a negative conversion), while 13 had a positive conversion of HER 2 status. In the 22 patients with a synchronous metastatic disease, 9 patients had a discordance in HER2 status. Six of these patients had a positive conversion, while three patients had a loss of HER2 amplification in the biopsy of the metastatic lesion. (Table 1)

25 of 28 with a discordant HER2 status were positive for ER and/or PgR in the primary tumor; respectively 8 of 9 in HER2 positive/negative and 17 of 19 in HER2 negative/positive cases. This was only in 20 of 46 patients in the HER2 concordant group. The median HER2/CEP17 ratios of the primary breast tumors were 2.5, 1.2 and 5.8 for respectively the HER2 positive/negative, HER2 negative/positive and HER2 positive/positive group. These median ratios of the metastatic tumors were 1.2, 2.5 and 5.5. (Table 2) In the group with a negative conversion, three patients had heterogeneity (globally interpreted as positive) on the FISH test of the primary lesion and two patients had also a metastasis with a positive HER2 status. (Appendix Table 1)

38 of the 46 cases received treatment with a taxane and trastuzumab in first or second line in the HER2 positive/positive group, of whom 13 received pertuzumab. In the HER2 positive/positive group 21 out of the 46 cases had a treatment with T-DM1; in the HER2 positive/negative group only 2 out of the 9 cases and in the HER2 negative/positive 7 out of the 19 cases. In the HER2 positive/positive group 27 out of the 46 cases had a treatment with capecitabine and lapatinib; in the HER2 positive/negative group only 2 out of 9 cases, and in the HER2 negative/positive group 7 out of the 19. (Table 2)

In patients with metachronous metastatic disease, the median global NPI score was 4.8; for HER2 positive/positive, HER2 positive/negative and HER2 negative/positive, respectively 5.0, 4.8 and 4.7. The median TTM was 45 months overall; with 35.50, 35.50 and 67 months, respectively for HER2 positive/positive, HER2 positive/negative and HER2 negative/positive cases. In the HER2 positive/positive group, 21 of the 33 cases with metachronous metastatic disease had a treatment with trastuzumab in the (neo-) adjuvant setting, while 5 of the 6 cases in the HER2 positive/negative group. Those patients had the diagnosis of the primary tumour before 2007. (Table 2)

\section{Progression-free survival}

Taxane with trastuzumab (+/- pertuzumab) 
The HER2 positive/positive group had a median PFS on taxane and trastuzumab (+/- pertuzumab) of 9.0 months (95\% CI 7.0 - 12.0), HER2 positive/negative 5.5 months (95\% CI 1.0 - 7.0) and the HER2 negative/positive of 14.0 months (95\% CI 2.0- not estimable). The log-rank analysis showed a significant effect of the different groups (uncorrected $\mathrm{p}=0.02$ and corrected $\mathrm{p}=0.02$ ). PFS for taxane and trastuzumab (and pertuzumab) was significantly better for HER2 negative/positive cases compared HER2 positive/negative (uncorrected $\mathrm{P}=0.02$ and corrected $\mathrm{p}=0.01$ ), and better for HER2 positive/positive compared to the HER2 positive/negative group (uncorrected $\mathrm{p}=0.03$ and corrected $\mathrm{p}=0.01$ ). There was no significant difference in PFS between the HER2 negative/positive and HER2 positive/positive group (uncorrected $\mathrm{p}=0.23$ and corrected $\mathrm{p}=0.43$ ). (Figure 2)

\section{Trastuzumab - emtansine (T-DM1)}

The median PFS on T-DM1 was 6.0 months (95\% CI 4.0 - 8.0) in the HER2 positive/positive group, 1.5 months $(95 \%$ CI $1.0-2.0)$ in the HER2 positive/negative group and 1 month (95\% CI $1.0-5.0)$ in the HER2 negative/positive group. There was a significant difference in PFS between groups (p-value global test=0.02). The HER2 positive/positive had a significantly better PFS compared to the HER2 positive/negative $(\mathrm{p}=0.01$ and HER2 negative/positive group $(\mathrm{p}=0.04)$. There was no significant difference between HER2 positive/negative and HER2 negative/positive ( $\mathrm{p}=0.63$ ). (Figure 2)

\section{Capecitabine and lapatinib}

The HER2 positive/positive group had a median PFS for a treatment with capecitabine and lapatinib of 4 months (95\% CI 2.0 - 7.0), the HER2 positive/negative group 1.5 months (95\% CI $1.0-2.0$ ) and the HER2 negative/positive group 2.0 months (95\% CI 1.0 - 5.0). There was no significant global effect on PFS. But pairwise comparisons showed a significant p-value for HER2 positive/negative group versus HER2 positive/positive group however, this should be interpreted very carefully in the presence of a non-significant global p-value. (Figure 2)

\section{Overall survival}

After correcting for possible confounders (presence of primary metastatic disease, negative sex-steroid receptor in the primary tumor, a high NPI score (> 5.4) and a short TTM ( $\leq 12$ months)), there was a significant association between the different groups and the OS (HR 0.79, 95\% CI $0.356-1.738$ ). The HER2 positive/negative group had a significantly worse survival rate, compared to the HER2 negative/positive (HR 0.15, 95\% CI 0.06- 0.38) and HER2 positive/positive groups (HR 0.19, 95\% CI $0.08-0.44)$. There was no significant difference in OS between the HER2 negative/positive group and HER2 positive/positive group. (Figure 3 )

\section{Discussion}


This study showed a total discordance rate in HER2 status of paired biopsies (negative and positive conversion), in patients treated with trastuzumab, in a (neo-) adjuvant or metastatic setting, of $37.8 \%$. Discordance in receptor status between primary biopsy and metastatic lesion is well documented in the current literature, with a reported HER2 discordance rate between $3 \%$ and $30 \%$. (7,8,11,14-17) Our results are from a highly selected group of trastuzumab treated patients with stage IV disease but the negative conversion rate in $16 \%$ of the patients is therefore compatible with existing literature. Possible explanations for discordance in both directions are given in Yang et al., such as presence of small subclones in primary tumor, genetic drift or clonal selection and changes in receptor status as a survival mechanism. (18-21)

In the population with metachronous metastatic disease the majority of cases with conversion to a negative HER 2 status ( 5 out of 6 patients) had a prior treatment with trastuzumab in (neo-) adjuvant setting. This suggest that treatment with trastuzumab may contribute to the biological mechanisms of loss of HER2 amplification. A large trial with 549 cases of Wang et al. has already provided new evidence that anti-HER2 treatment has a significant impact on HER2 loss, more over HER2 loss was more frequently shown in patients treated with paclitaxel, carboplatin, and trastuzumab (19.8\%), compared to patients treated with paclitaxel, carboplatin only (9.4\%). (22)

In the present literature, there is no data available supporting the choice of therapy in cases with HER2 discordance between primary and metastatic tumor. The ESMO guidelines recommend considering the use of targeted therapy, if hormone receptors or HER2 status were positive at least once. (23) Amir et $a l$. evaluated whether results of biopsy of metastatic lesion altered management in patients with discordant receptor status; gain of HER2 amplification in biopsy of metastatic disease with addition of trastuzumab was seen in 6 of the 73 cases. (5) The ASCO guidelines also recommended that in patients with discordance of results between primary and metastatic tissues, the Panel consensus is to use preferentially the ER, PgR, and HER2 status of the metastasis to direct therapy if supported by the clinical scenario and patient's goals for care. Although the Panel recognizes that there is sufficient evidence for biomarker change from primary to metastasis, there is no evidence to demonstrate that systemic therapy choices affect health outcomes when biomarker change occurs. (24)

To the best of our knowledge, our study is the first evaluating PFS using HER2 inhibitors and chemotherapy in metastatic breast cancer by HER 2 concordance. Our results show that patients with a negative conversion of the HER2 status had a significantly shorter PFS using HER2 inhibitors and chemotherapy, compared to patients with concordant HER2 status. These findings are significant for the combined therapy taxane/trastuzumab (+/- pertuzumab) and T-DM1, but insignificant for a treatment with lapatinib and capecitabine. Although there is a significant difference with a treatment with T-DM1, we have to take into account that the sample size was very small. We can conclude that loss of amplification seems to have a negative predictive value for PFS using taxane/trastuzumab (+/- 
pertuzumab) and T-DM1. For cases with a positive conversion of the HER2 status on the biopsy of the metastatic lesion, the efficacy of HER2 inhibitors was similar for PFS using taxane/trastuzumab (+/pertuzumab) compared to the concordant group. However, the median PFS in the HER2 negative/positive group was 14 months compared to 9 months in the concordant group. These results clearly do suggest that cases with positive HER2 conversion in a biopsy of metastatic breast cancer do benefit from taxane/trastuzumab (+/- pertuzumab). Nevertheless, these data need to be confirmed in larger series. Remarkably, PFS using T-DM1 was significantly lower for cases with a positive HER2 conversion compared to the concordant group. Five of the seven cases had no benefit at all. So, in our small series it seems that gain of HER2 amplification was not predictive for benefit on T-DM1. Although our data suggests that cases with a positive conversion of HER2 status might predict for T-DM1 resistance, data from larger series are needed and, to our knowledge, aren't reported as such in the available T-DM1 literature.

In the group with a negative conversion, three patients have a heterogeneity (globally interpreted as positive) on the FISH test of the primary lesion and two patients had also a metastasis with a positive HER2 status. Tumor heterogeneity is already known for a longer time, although the exact role in tumor progression and therapeutic response stays unclear. (25) Since the introduction of $89 \mathrm{Zr}$ Trastuzumab PET/CT heterogeneity in HER2 status is increasingly being recognized. The ZEPHIR clinical trial showed that pretreatment imaging of HER2 withHER2-PET/CT is a promising tool for studying interlesion heterogeneity in advanced disease stages. When combined with early FDGPET/CT after one cycle of T-DM1, it was powerful in predicting which patients will or will not benefit from T-DM1. (26) Our study highlighted the importance of the role of knowledge of the HER2 status in the metastatic lesion. It can be discussed whether it is best performed by taking biopsy of the metastatic lesion, or by doing a HER2 PET/CT scan. Nowadays, we advise to take a biopsy of the metastatic lesion, although it is only a small sample of the tissue, furthermore lesions are not always accessible. In the future the introduction of the HER2 PET/CT has a great potential to play a major role to determine HER2 status.

In literature, some contradiction about OS in HER2 discordant groups exists. Most analyses suggested that discordance in hormone or HER2 receptor status is associated with a poorer survival rate.

$(5,7,12,13)$ However, the study of Amir et al. showed no difference in OS if treatment was modified according to the biopsy of metastatic lesion. After correcting for hormone receptor status, NPI score, primary metastasis and TTM, our results showed that patients with a negative HER2 conversion had a significantly shorter OS compared to the concordant group and the cases with positive HER2 conversion. The significantly shorter PFS using HER2 inhibitors in the HER2 positive/negative cases could contribute to this result. The group with a positive conversion of the HER2 status had a similar OS compared to the concordant group. 
Our study has some limitations. First of all, its small sample size and retrospective nature. Larger series and prospective studies should be performed to confirm our findings. Secondly, it would also be interesting to have a HER2 positive/negative control group that did not receive anti-HER2 directed therapy. Not all primary samples, taken in other centres, no central pathology review was performed, therefore difference in the interpretation of the HER2 IHC might have occurred being a source of interobserver variability. In addition, there may have been some selection bias because second biopsies may have been collected more often in patients not responding well to anti-HER2 therapy in general. Finally, there was no correction for possible confounders for determination of PFS for different treatments due to the small simple size.

Given that HER2 discordance in stage IV breast cancer had an impact on PFS and OS, we stress the importance of taking a biopsy of the metastatic lesion; HER2 PET/CT imaging has potentials. In the meantime, we suggest trastuzumab and taxane (+/- pertuzumab) in cases with a positive conversion of the HER2 status in the metastatic lesion. A possible solution is a prospective trial to confirm our data and determine whether adapting targeted therapy based on the new receptor status of the metastatic lesion might improve patient outcome.

\section{Conclusion}

Conversion of HER 2 status was seen in 28 out of 74 cases and was mostly observed in hormone receptorpositive tumors. Patients with a positive conversion of HER2 derive substantial benefit from first line treatment including HER2 inhibitors. In this series, loss of HER2 amplification was predictive for poor treatment response using anti-HER2 therapies. This retrospective study underlines the importance of taking a biopsy of the metastatic lesion and adapt therapy based on the result of the HER2 receptor status of the metastasis.

\section{Conflict of interest}

The authors declare that they have no conflict of interest.

\section{Ethical approval}

All procedures performed in studies involving human participants were in accordance with the ethical standards of the institutional and/or national research committee and with the 1964 Helsinki declaration and its later amendments or comparable ethical standards. For this type of retrospective study, formal consent is not required. The ethics committee of the University Hospitals Leuven approved the study.

\section{Figures and tables}


Fig. 1 CONSORT-flow diagram

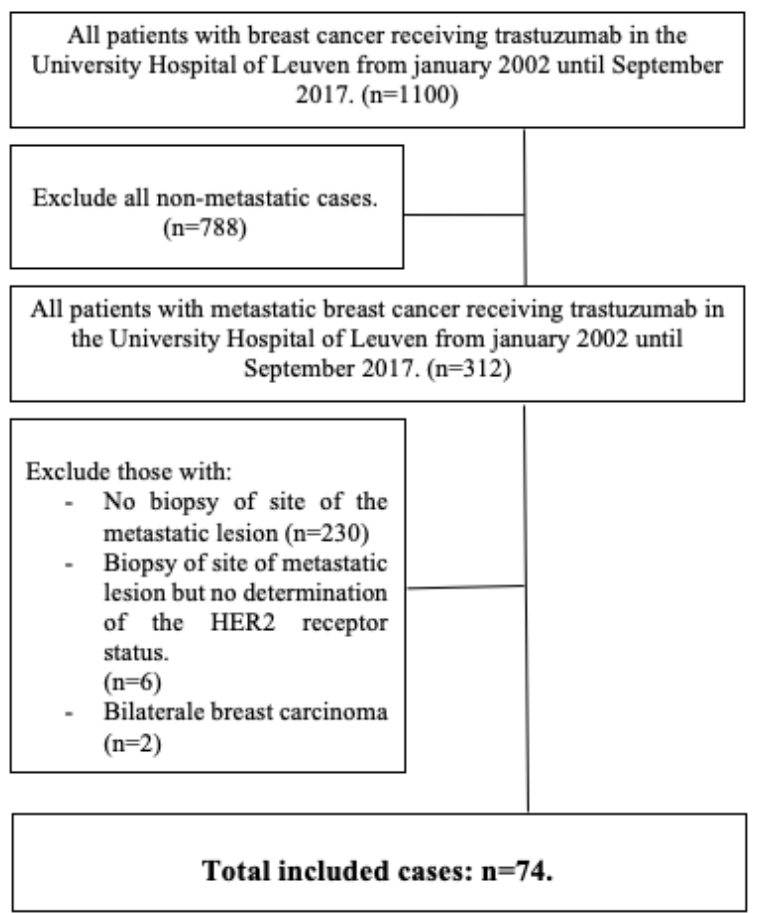

Table 1 Absolute numbers of cases with concordance and discordance in HER2 status

\begin{tabular}{lll}
\hline $\begin{array}{l}\text { HER2 status primary biopsy/biopsy metastatic } \\
\text { lesion }\end{array}$ & $\begin{array}{l}\text { Synchronous } \\
\text { metastatic disease } \\
(\mathbf{n = 2 2})\end{array}$ & $\begin{array}{l}\text { Metachronous } \\
\text { metastatic disease } \\
(\mathbf{n = 5 2})\end{array}$ \\
\hline HER2 positive/positive & 13 & 33 \\
HER2 positive/negative & 3 & 6 \\
HER2 negative/positive & 6 & 13 \\
\hline
\end{tabular}

Table 2 General patients' characteristics

\begin{tabular}{|c|c|c|c|c|}
\hline \multirow{3}{*}{ Subgroup } & & HER2 pos./pos. & HER2 pos./neg. & HER2 neg./pos. \\
\hline & & \multicolumn{3}{|c|}{ No } \\
\hline & & 46 & 9 & 19 \\
\hline $\begin{array}{l}\text { Median Age diagnosis } \\
\text { metastatic disease } \\
\text { (years) }\end{array}$ & & $53.5(36-90)$ & $57(23-77)$ & $53(43-11)$ \\
\hline $\begin{array}{l}\text { Synchronous metastatic } \\
\text { disease }\end{array}$ & & $28.2 \%(\mathrm{n}=13)$ & $27.2 \%(\mathrm{n}=3)$ & $31.6 \%(\mathrm{n}=6)$ \\
\hline \multirow[t]{2}{*}{$\begin{array}{l}\text { Metachronous } \\
\text { metastatic disease }\end{array}$} & & 33 & 6 & 13 \\
\hline & $\begin{array}{l}\text { - Median TTM (months) } \\
\text { - Median NPI score } \\
\text { - Trastuzumab in a (neo-) } \\
\text { adjuvant setting }\end{array}$ & $\begin{array}{c}35.5 \\
5 \\
21\end{array}$ & $\begin{array}{c}35.5 \\
4.8 \\
5\end{array}$ & $\begin{array}{c}67.0 \\
4.7 \\
0\end{array}$ \\
\hline $\begin{array}{l}\text { Positive ER or/and PgR } \\
(\%)\end{array}$ & & $43.4 \%(n=20)$ & $88.8 \%(\mathrm{n}=8)$ & $94.7 \%(\mathrm{n}=17)$ \\
\hline Type of tumor & - Ductal & $5.5 \%(\mathrm{n}=43)$ & $100 \%(\mathrm{n}=9)$ & $79.9 \%(n=14)$ \\
\hline
\end{tabular}




\begin{tabular}{|c|c|c|c|c|}
\hline & - Lobular & $93.4 \%(n=3)$ & 1 & $26.3 \%(\mathrm{n}=5)$ \\
\hline \multirow[t]{2}{*}{ Grade } & - Grade 2 & $37 \%(\mathrm{n}=17)$ & $66.7 \%(\mathrm{n}=6)$ & $31.6 \%(\mathrm{n}=6)$ \\
\hline & - Grade 3 & $60.8 \%(\mathrm{n}=28)$ & $33.3 \%(n=3)$ & $86.4 \%(n=13)$ \\
\hline \multirow{2}{*}{$\begin{array}{l}\text { Median HER2/CEP17 } \\
\text { ratio }\end{array}$} & - Breast & 5.8 & 2.5 & 1.2 \\
\hline & - Metastatic lesion & 5.5 & 1.2 & 2.5 \\
\hline \multirow[t]{3}{*}{ Anti-HER2 therapy } & $\begin{array}{l}\text { - Taxane and trastuzumab } \\
(+ \text { pertuzumab) } \\
\left.\text { (in } 1^{\text {st }} \text { or } 2 \text { th line }\right)\end{array}$ & $38(12)$ & $8(4)$ & $10(7)$ \\
\hline & - T-DM1 & 21 & 2 & 7 \\
\hline & $\begin{array}{l}\text { - Capecitabine and } \\
\text { lapatinib }\end{array}$ & 27 & 2 & 7 \\
\hline
\end{tabular}


1) Taxane with trastuzumab ( $+/$ - pertuzumab)
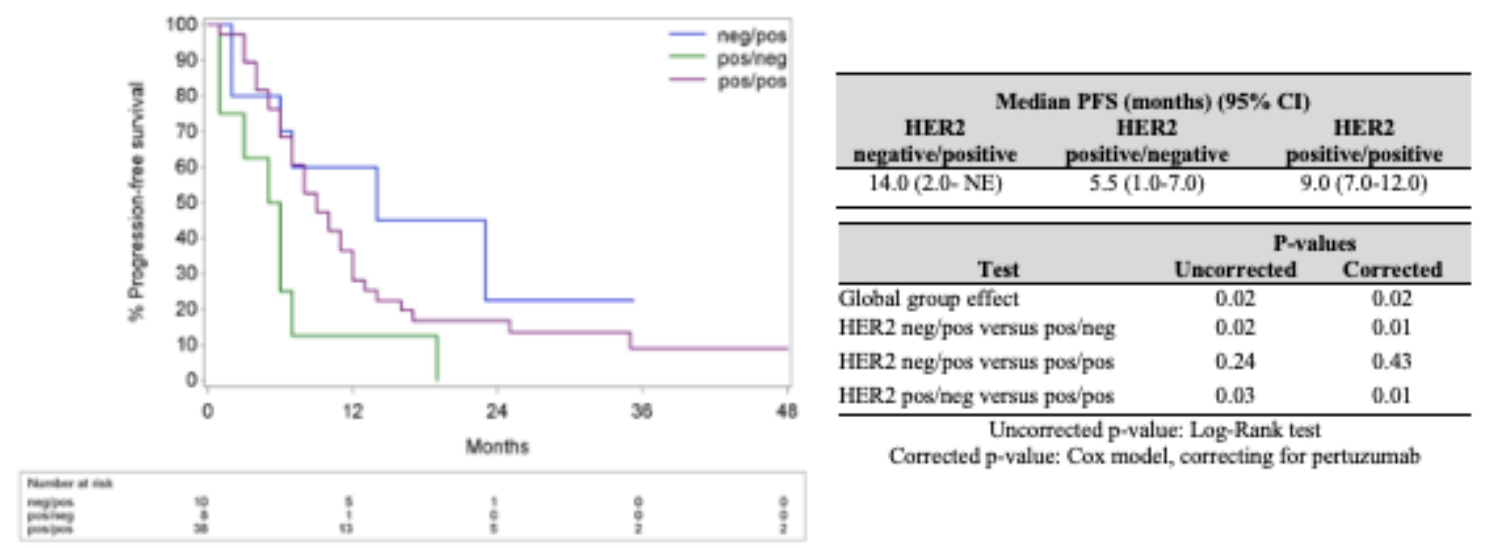

2) T-DM1
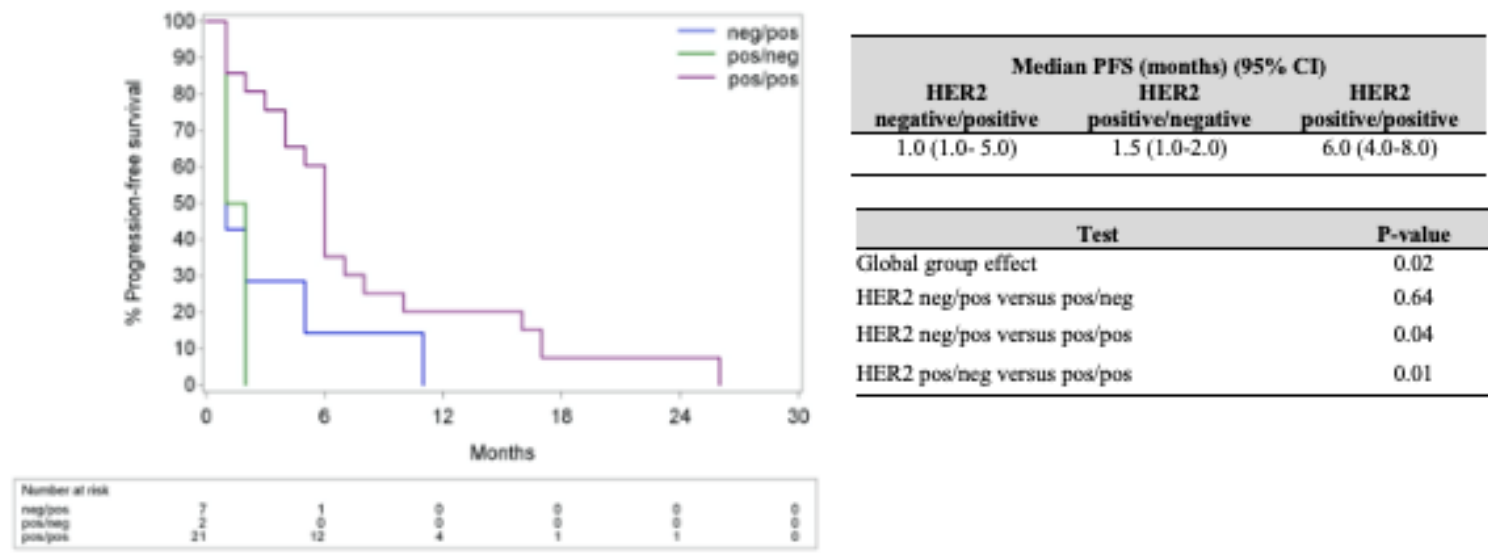

3) Capecitabine with lapatinib

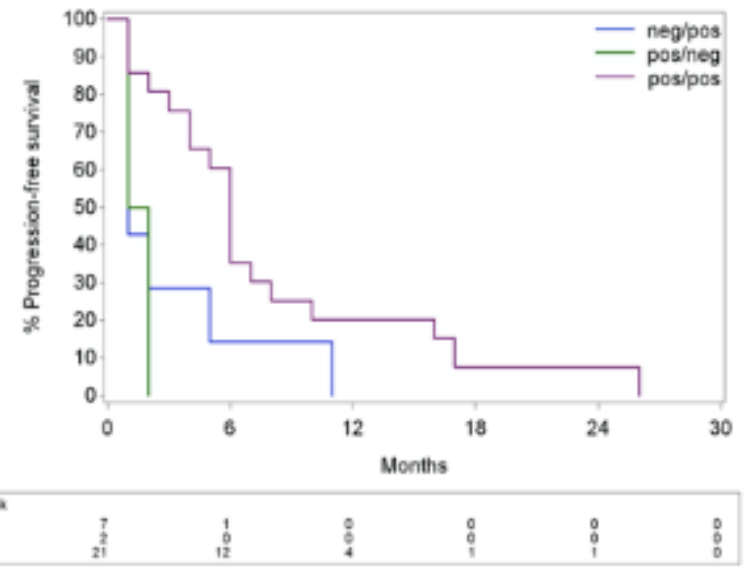

\begin{tabular}{|c|c|c|}
\hline \multicolumn{3}{|c|}{ Median PFS (months) (95\% CI) } \\
\hline $\begin{array}{c}\text { HER2 } \\
\text { nezative/positive }\end{array}$ & $\begin{array}{c}\text { HER2 } \\
\text { positive'negative }\end{array}$ & $\begin{array}{c}\text { HER2 } \\
\text { positive/positive }\end{array}$ \\
\hline $2.0(1.0-5.0)$ & $1.5(1.0-2.0)$ & $4.0(2.0-7.0)$ \\
\hline & Test & P-value \\
\hline Global group effect & & 0.90 \\
\hline HER2 neg/pos versus & os/neg & 0.18 \\
\hline HER2 neg/pos versus & os/pos & 0.35 \\
\hline HER2 pos/beg versus & os/pos & 0.04 \\
\hline
\end{tabular}

Fig. 2 Kaplan-meier curve for respectively taxane with trastuzumab (1), T-DMI (2) and capecitabine with lapatinib (3) according to HER2 negative/positive (neg/pos, blue curve), HER2 positive'negative (pos/neg, green curve) and HER2 positive/positive (pos/pos, purple curve). 


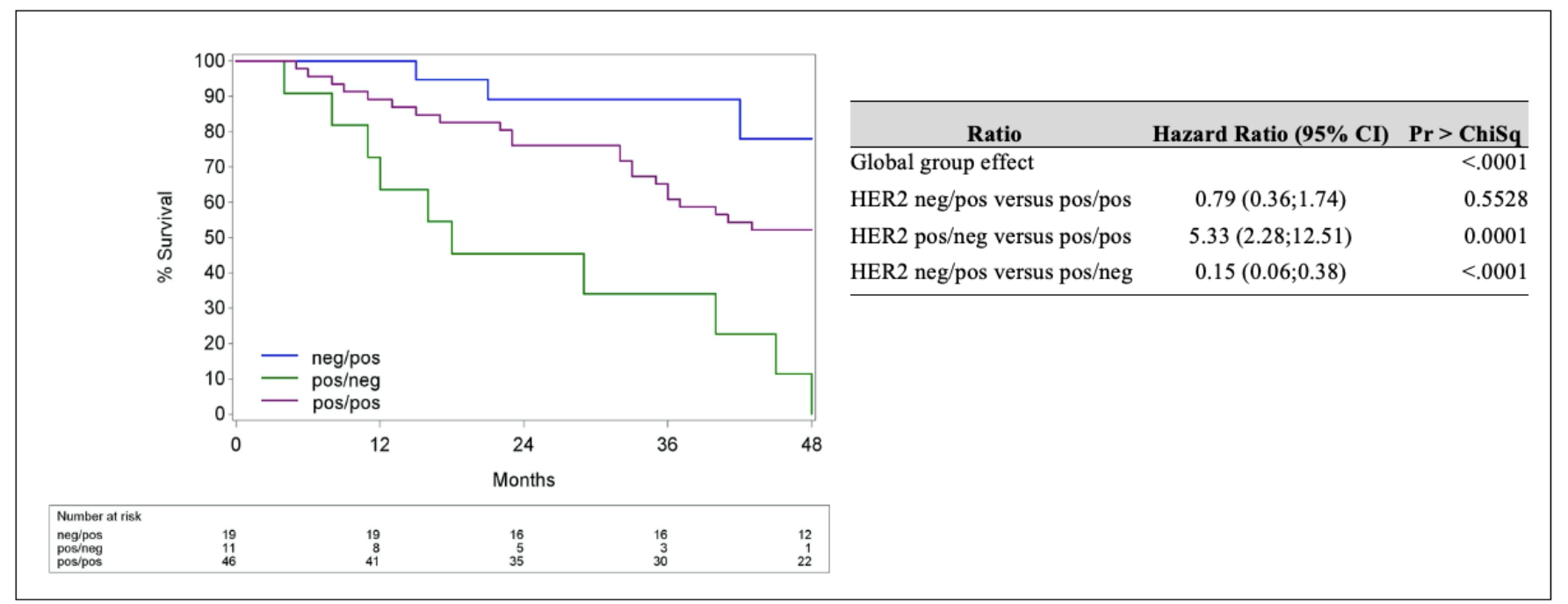

Fig. 3 Kaplan-meier curve for overall survival according to HER2 negative/positive (neg/pos, blue curve), HER2 positive/negative (pos/neg, green curve) and HER2 positive/positive (pos/pos, purple curve).

\section{Appendix}

Table 1 patients' characteristics

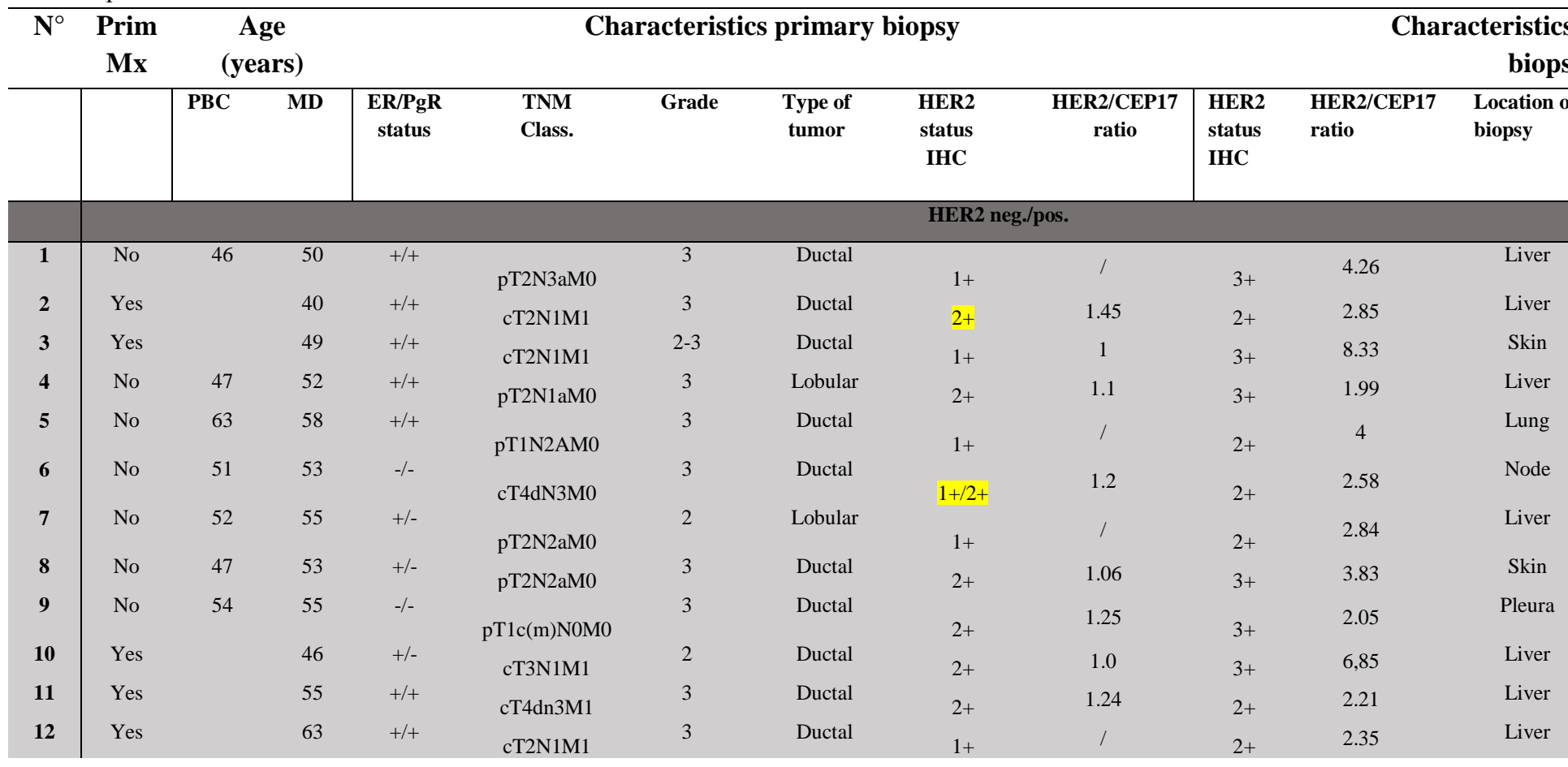




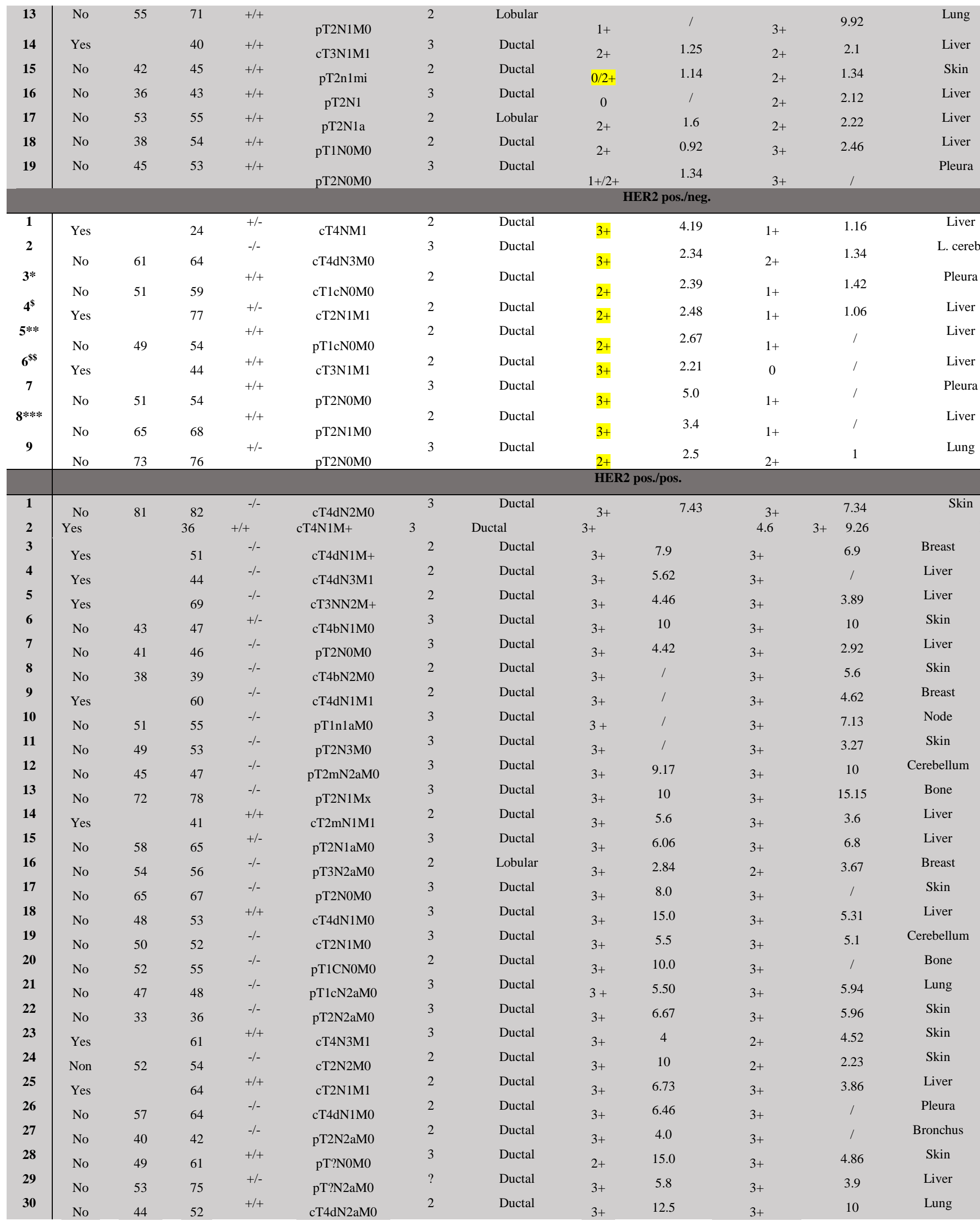




\begin{tabular}{|c|c|c|c|c|c|c|c|c|c|c|c|c|}
\hline 31 & No & 38 & 50 & $-1-$ & pT1N0M0 & 3 & Ductal & $3+$ & 5.8 & $3+$ & 9.57 & Skin \\
\hline 32 & No & 41 & 43 & $+/+$ & pT2N0M0 & 2 & Lobular & $3+$ & 5 & $3+$ & 3.67 & Lung \\
\hline 33 & No & 67 & 70 & $-/-$ & cT4dN2M0 & 3 & Ductal & $3+$ & 4.76 & $3+$ & I & Pleura \\
\hline 34 & Yes & & 50 & $+/+$ & cT3N1M1 & $2-3$ & Ductal & $3+$ & 5.38 & $3+$ & I & Liver \\
\hline 35 & No & 55 & 59 & $+/+$ & pT2N0M0 & 3 & Ductal & $3+$ & 5.0 & $2+$ & 7.5 & Pleura \\
\hline 36 & Yes & & 63 & $+/+$ & cT2N0M1 & 3 & Ductal & $3+$ & 6.74 & $3+$ & 7.3 & Skin \\
\hline 37 & No & 51 & 54 & $-/-$ & cT4dN1M0 & 3 & Ductal & $3+$ & & $3+$ & 7.67 & Pleura \\
\hline 38 & Yes & & 49 & $+/+$ & cT2N1M1 & 3 & Ductal & $3+$ & 8.24 & $3+$ & I & Liver \\
\hline 39 & No & 75 & 76 & $+/+$ & pT3N3aM0 & 2 & Ductal & $3+$ & 4.21 & $3+$ & 3.80 & Stomach \\
\hline 40 & No & 46 & 49 & $+/-$ & pT2N2aM0 & 2 & Ductal & $2+$ & 4.2 & $3+$ & 2.41 & Liver \\
\hline 41 & No & 45 & 59 & $+/-$ & cT4dN2M0 & 3 & Lobular & $2+$ & & $3+$ & 7.89 & Brain \\
\hline 42 & No & 71 & 73 & $+/+$ & pT1CN1aM0 & 3 & Ductal & $3+$ & 5.0 & $3+$ & I & Liver \\
\hline 43 & Yes & & 73 & $-1-$ & cT4dN1M1 & 2 & Ductal & $3+$ & 5.70 & $2+$ & 2.18 & Liver \\
\hline 44 & Yes & & 44 & $-/-$ & cT3N1M3 & 3 & Ductal & $3+$ & 15.0 & $3+$ & 4.74 & Skin \\
\hline 45 & No & 33 & 35 & $+/+$ & pT2NOM0 & 3 & Ductal & $3+$ & I & $3+$ & 1 & Liver \\
\hline 46 & No & 42 & 44 & $-/-$ & pT2NOM0 & 3 & Ductal & $3+$ & 8.34 & $3+$ & 6.25 & Skin \\
\hline
\end{tabular}

PBC: primary breastcancer, MD: metastatic disease, Prim Mx: primary metastatic disease, pert: pertuzumab, trast: trastuzumab, cap: capecitabine and lap: lapatinib.

* Heterogeneity: biopsy of skin metastasis was positive.

${ }^{\$}$ Heterogeneity: heterogeneity on the FISH test of the primary lesion. Majority of the cells 5-6 HER2 signals or ratio > 2, globally interpreted as FISH positive.

** Heterogeneity: metastasis on the rib was IHC $3+$.

\$ Heterogeneity on the FISH test of the primary lesion. Majority of the cells 5-6 HER2 signals or ratio > 2, globally interpreted as FISH positive.

${ }^{\star * \star}$ Heterogeneity on the FISH test of the primary lesion. Majority of the cells 5-6 HER2 signals or ratio > 2, globally interpreted as FISH positive.

\section{References}

ADDIN Mendeley Bibliography CSL_BIBLIOGRAPHYX1. Slamon DJ, Godolphin W, Jones LA, Holt JA, Wong SG, Keith DE, Levin WJ, Stuart SG, Udove J UA. Studies of the HER2/neu proto-oncogene in human breast and ovarian cancer. Science May 1989, 244(707-12).

2. Ross JS, Slodkowska EA, Symmans WF, Pusztai L, Ravdin PM, Hortobagyi GN. The HER-2 Receptor and Breast Cancer: Ten Years of Targeted Anti-HER-2 Therapy and Personalized Medicine. Oncologist. 2009;14(4):320-68.

3. Yao Z, Lu L, Wang R, Jin L, Liu S, Li H, et al. Discordance and clinical significance of ER, PR, and HER2 status between primary breast cancer and synchronous axillary lymph node metastasis. Med Oncol. 2013; 31(1):798.

4. Gajria D, Chandarlapaty S. HER2-amplified breast cancer: mechanisms of trastuzumab resistance and novel targeted therapies. Expert Rev Anticancer Ther. 2011 Feb; 11(2):263-75.

5. Amir E, Miller N, Geddie W, Freedman O, Kassam F, Simmons C, et al. Prospective Study Evaluating the Impact of Tissue Confirmation of Metastatic Disease in Patients With Breast Cancer. J Clin Oncol. 2012 Feb 20; 30(6):587-92.

6. Thompson AM, Jordan LB, Quinlan P, Anderson E, Skene A, Dewar JA, et al. Prospective comparison of switches in biomarker status between primary and recurrent breast cancer: the Breast Recurrence In Tissues Study (BRITS). Breast Cancer Res. 2010 Nov 8; 12(6):R92-R92. 
7. Pareja F, Murray MP, Jean R Des, Konno F, Friedlander M, Lin O, et al. Cytologic assessment of estrogen receptor, progesterone receptor, and HER2 status in metastatic breast carcinoma. $\mathbf{J}$ Am Soc Cytopathol. 2017; 6(1):33-40.

8. Aurilio G, Disalvatore D, Pruneri G, Bagnardi V, Viale G, Curigliano G, et al. A meta-analysis of oestrogen receptor, progesterone receptor and human epidermal growth factor receptor 2 discordance between primary breast cancer and metastases. Eur J Cancer. 2014; 50(2):277-89.

9. Willemijne A M E Schrijver, Karijn P M Suijkerbuijk, Carla H van Gils, Elsken van der Wall, Cathy B Moelans, Paul J van Diest et al. Receptor Conversion in Distant Breast Cancer Metastases: A Systematic Review and Meta-analysis Journal of the National Cancer Institute, Volume 110, Issue 6, June 2018, Pages 568-580.

10. Van Poznak C, Somerfield MR, Bast RC, Cristofanilli M, Goetz MP, Gonzalez-Angulo AM, et al. Use of Biomarkers to Guide Decisions on Systemic Therapy for Women With Metastatic Breast Cancer: American Society of Clinical Oncology Clinical Practice Guideline. J Clin Oncol. 2015 Aug 20;33(24):2695-704.

11. Amir E, Clemons M, Purdie CA, Miller N, Quinlan P, Geddie W, et al. Tissue confirmation of disease recurrence in breast cancer patients: Pooled analysis of multi-centre, multi-disciplinary prospective studies. Cancer Treat Rev. 2012; 38(6):708-14.

12. Wilking U, Karlsson E, Skoog L, Hatschek T, Lidbrink E, Elmberger G, et al. HER2 status in a population-derived breast cancer cohort: discordances during tumor progression. Breast Cancer Res Treat. 2011; 125(2):553-61.

13. Liedtke C, Broglio K, Moulder S, Hsu L, Kau S-W, Symmans WF, et al. Prognostic impact of discordance between triple-receptor measurements in primary and recurrent breast cancer. Ann Oncol. 2009 Dec 12; 20(12):1953-8.

14. Lower EE, Khan S, Kennedy D, Baughman RP. Discordance of the estrogen receptor and HER$2 /$ neu in breast cancer from primary lesion to first and second metastatic site. Breast Cancer (London). 2017 Aug 2;9:515-20.

15. Kulka J, Székely B, Lukács L V, Kiss O, Tökés A-M, Vincze E, et al. Comparison of Predictive Immunohistochemical Marker Expression of Primary Breast Cancer and Paired Distant Metastasis using Surgical Material: A Practice-Based Study. J Histochem Cytochem. 2016 Apr 30;64(4):256-67.

16. Li MH, Hou CL, Wang C, Sun AJ. HER-2, ER, PR status concordance in primary breast cancer and corresponding metastatic lesion in lymph node in Chinese women. Pathol - Res Pract. 2016;212(4):252-7.

17. Lindström LS, Karlsson E, Wilking UM, Johansson U, Hartman J, Lidbrink EK, et al. Clinically Used Breast Cancer Markers Such As Estrogen Receptor, Progesterone Receptor, and Human Epidermal Growth Factor Receptor 2 Are Unstable Throughout Tumor Progression. J Clin Oncol. 2012 Jul 20; 30(21):2601-8.

18. Yang Y-F, Liao Y-Y, Yang M, Peng N-F, Xie S-R, Xie Y-F. Discordances in ER, PR and HER2 receptors between primary and recurrent/metastatic lesions and their impact on survival in breast cancer patients. Med Oncol. 2014; 31(10):214.

19. van de Ven S, Smit VTHBM, Dekker TJA, Nortier JWR, Kroep JR. Discordances in ER, PR and HER2 receptors after neoadjuvant chemotherapy in breast cancer. Cancer Treat Rev. 2011;37(6):422-30.

20. Dowsett M, Dunbier AK. Emerging Biomarkers and New Understanding of Traditional Markers in Personalized Therapy for Breast Cancer. Clin Cancer Res. 2008 Dec 15;14(24):8019 LP-8026.

21. Perou CM, Sørlie T, Eisen MB, van de Rijn M, Jeffrey SS, Rees CA, et al. Molecular portraits of human breast tumours. Nature. 2000 Aug 17;406:747.

22. Ruo-Xi Wang, Sheng Chen 34 , Xi Jin 1 2, Can-Ming Chen 1 2, Zhi-Ming Shao 5 Weekly paclitaxel plus carboplatin with or without trastuzumab as neoadjuvant chemotherapy for HER2positive breast cancer: loss of HER2 amplification and its impact on response and prognosis. Breast Cancer Res Treat 2017 Jan;161(2):259-267.

23. Cardoso F, Fallowfield L, Costa A, Castiglione M, Senkus E, ESMO Guidelines Working Group $\mathrm{O}$ behalf of the EGW. Locally recurrent or metastatic breast cancer: ESMO Clinical Practice Guidelines for diagnosis, treatment and follow-up. Ann Oncol. 2011;22 Suppl 6(suppl 6):vi2530 . 
24. Catherine Van Poznak, Mark R. Somerfield, Robert C. Bast, et al. Use of Biomarkers to Guide Decisions on Systemic Therapy for Women With Metastatic Breast Cancer: American Society of Clinical Oncology Clinical Practice Guideline J Clin Oncol. 2015 Aug 20; 33(24): 26952704.

25. Nicholas McGranahan ${ }^{1}$, Charles Swanton ${ }^{2}$ et al. Biological and therapeutic impact of intratumor heterogeneity in cancer evolution Cancer Cell 2015 Jan 12;27(1):15-26. doi: 10.1016/j.ccell.2014.12.001.

26. G.Gebhart ${ }^{1}$ L.E.Lamberts ${ }^{2}$ Z.Wimana ${ }^{1}$ et al. Molecular imaging as a tool to investigate heterogeneity of advanced HER2-positive breast cancer and to predict patient outcome under trastuzumab emtansine (T-DM1): the ZEPHIR trial Annals of Oncology Volume 27, Issue 4, April 2016, Pages 619-624 